\title{
Impact of the vertical discretization of analysis data on the estimates of atmospheric inertio-gravity energy
}

\author{
N. Žagar*, \\ University of Ljubljana and Center of Excellence "SPACE-SI", Ljubljana, Slovenia, \\ K. Terasaki and H. L. Tanaka \\ University of Tsukuba, Tsukuba, Japan
}

*Corresponding author address: Faculty of Mathematics and Physics, Jadranska 19, 1000 Ljubljana, Slovenia. E-mail: nedjeljka.zagar@fmf.uni-lj.si 


\begin{abstract}
This paper compares energy percentages of the large-scale inertio-gravity waves in the atmosphere obtained from the analysis data on model levels and standard pressure levels. Two methods for solving the vertical structure equation are applied. The results show that different methods lead to the same result when and that the model-level data provide more detailed information about the tropical inertiogravity circulations in atmospheric layers not resolved by the standard pressurelevel data. The estimated level of the inertio-gravity wave energy on large scales is about $10 \%$ of the global wave energy.
\end{abstract}




\section{Introduction}

The role of inertio-gravity (IG) energy in atmospheric energetics on various scales is still uncertain. First of all, a precised quantitative separation of the balanced (i.e. nearly geostrophic or Rossby-type) and IG (unbalanced) circulation from observational data is difficult. At the same time, (re)analysis data used to understand the IG circulation have their main shortcomings at the mesoscale and in the Tropics, regions where the IG flows matter the most. Uncertainties are associated with difficulties in atmospheric data assimilation in these regimes and the lack of observations, espcially the wind observations (e.g. Žagar et al. 2004, 2005).

Early study by Daley (1983) estimated that the average percentage of ageostrophic motions in early analyses of ECMWF was about $10 \%$; this implies that about $1 \%$ of atmospheric wave energy is associated with unbalanced flows. Compared to the early analyses which described tropical flows rather poorly (e.g. Heckley 1985), present-day analysis datasets are considerably better thanks to large amounts of satellite observations assimilated, to improved assimilation methodology and to better models (e.g. Simmons and Hollingsworth 2002). In particular, model resolution has increased (in winter 2011 the ECMWF system has horizontal resolution T1279 which corresponds to about $16 \mathrm{~km}$ grid) and precipitation forecast improved (e.g. Andersson et al. 2005).

A better description of IG circulations on the mesoscale, in the middle atmosphere and in the Tropics implies that the percentage of IG energy is different from $1 \%$; i.e. the percentage should be larger. Accordingly, Žagar et al. (2009a, 2010) reported that the level of IG energy in the large-scale wave motions in current analysis systems is about $10 \%$. Nearly the same number was obtained for the ECMWF and the NCEP operational analyses in spite of their different model depth (80 km and $60 \mathrm{~km}$ in the case of ECMWF and NCEP, respectively). The obtained percentage is about three times larger than the previous estimate by Tanaka and Kimura (1996) (hereafter TK1996) based on about 15 years older analysis systems. While it was impossible to precisely identify reason(s) for the different result, Žagar et al. (2009a) (hereafter ZTAR2009) argued as possible reasons different methodology of the applied normal-mode representation and properties of datasets which reflect advancements in the quality of atmospheric analyses in the Tropics. 
Both ZTAR2009 and TK1996 applied the normal-mode function (NMF) expansion to divide energy into the balanced and IG parts. The present note compares the two NMF expansion procedures to show that the energy percentages obtained by the two methods are the same when the same input data with high vertical resolution are used.

The paper is organized as follows. In Section 2 we briefly present the main difference between methodologies used in ZTAR2009 and TK1996 and we describe performed sensitivity experiments. Results are presented in Section 3 and conclusions are stated in Section 4.

\section{Estimates of atmospheric energetics}

\section{a. Solution of the vertical structure equation}

The derivation of NMFs relies on the assumption that the solutions to the linearized primitive equations can be expressed as a product of a horizontally independent part and horizontal eigensolutions. The horizontal and vertical structures are coupled through the separation constant, the equivalent depth. The expansion of three-dimensional atmospheric data into normal modes starts with the vertical projection followed by the horizontal expansion for each equivalent depth. The horizontal expansion functions are analytical solution of the linearized shallowwater equation on the sphere, called the Hough functions (Kasahara 1976). The vertical structure functions can be obtained analytically only for some special cases such as the isothermal atmosphere (Daley 1991, Chapter 6) or a constant stability profile (Terasaki and Tanaka 2007). For realistic temperature and stability profiles, solutions need to be obtained numerically.

The solution of the vertical structure equation in the $\sigma$ system obtained by Kasahara and Puri (1981) applies the top and bottom boundary conditions that the vertical velocity vanishes. Input data are in this case presented on $\sigma$-levels and temperature and stability profiles use globally average values on $\sigma$ surfaces. Kasahara and Puri (1981) applied the finite-difference method resulting in zigzag shapes of the vertical structure functions. The same approach was followed in ZTAR2009. The discussion of the accuracy of numerical solutions in $\sigma$ coordinates is provided Staniforth et al. (1985). Tanaka (1985), Tanaka and Kung (1988) and TK1996 applied the finite difference method with the pressure vertical coordinate and the bottom boundary condition that the surface wind vanishes. 
The second approach for solving the vertical structure equation numerically is the spectral approach developed by Kasahara (1984). In this case, the solution involves the use of the Legendre polynomials, derivatives in the equation are computed analytically and the Galerkin procedure is applied to determine the expansion coefficients. The vertical levels are located on the Gaussian grid which corresponds to the input pressure values divided by the surface pressure. For details, see Appendix of Kasahara (1984). The top boundary condition is that $\omega$ vanishes at $p=0$. The bottom boundary condition requests that the linearized version of the vertical velocity $d z / d t$ vanishes at the constant pressure $p_{s}$ near the surface.

Both the finite difference and the spectral approach result in the shapes of vertical structure functions with significant amplitude at levels close to the model top for the leading modes. The amplitude moves downward towards the surface as the vertical mode index increases. This is illustrated in Fig. 1 for the 91-level discretization of the operational ECMWF model. The main difference in the appearence of solutions is that the finite-difference solution for higher vertical modes have many zero crossings which are nearly identical to zero value (Fig. 1a) while the spectral solution for higher modes have small amplitudes also in the upper part of the model domain (Fig. 1b).

\section{b. Sensitivity experiments}

Sensitivity experiments are based on the comparison of the vertical discretization of input analysis data for the normal-mode projection. Two methods for solving the vertical structure equation are applied to the state-of-the-art analysis fields. The data are operational analyses of ECMWF for July 2007 which are result of the 12-hour window 4DVAR assimilation scheme and the model cycle Cy32r2. The original horizontal data resolution was defined by the horizontal truncation T799 $(\sim 25 \mathrm{~km})$ but the data used in ZTAR2009 and in this study were interpolated to a regular (Gaussian N64) grid with 256 and 128 points in the longitudinal and latitudinal directions, respectively. The reference experiment is the one presented in ZTAR2009 that applies 91 vertical model-level data with a model top level located at $0.01 \mathrm{hPa}$. Two sensitivity experiments with different vertical discretization are performed by using the spectral solution method of the vertical structure equation. The first experiment uses the same 91 model-level data as ZTAR2009. The second sensitivity experiment uses input data at standard pressure lev- 
els as in TK1996. The pressure-level data were extracted directly from the ECMWF retrieval system (MARS) and they include 21 standard pressure levels distributed between $1000 \mathrm{hPa}$ and $1 \mathrm{hPa}$ : 11 levels up to $100 \mathrm{hPa}, 5$ levels between 100 and $10 \mathrm{hPa}$ and 5 levels above $5 \mathrm{hPa}$. For comparison, TK1996 used 12 standard pressure levels up to $50 \mathrm{hPa}$ and the horizontal grid $2.5 \times 2.5$ degrees.

The reference experiment of ZTAR2009 interpolated 91 hybrid $(\sigma-p)$ model levels onto their equivalent $\sigma$ levels which was followed by the finite difference solution of the vertical structure equation and the horizontal projection for each equivalent depth. We denote this experiment as M91FD. An additional experiment performed in the same way excluded top 11 model levels (mesosphere) and started from the model level 12, located at about $1.8 \mathrm{hPa}$ (experiment M80FD). Since its results are basically the same as those for the M91FD experiment, it is not especially discussed. The two experiments applying the spectral method are denoted M91SP and P21SP. The earlier interpolates 91 model-level data to a 90-level Gaussian (pressure) grid with the top level located at $0.18 \mathrm{hPa}$. The latter experiment makes use of the 21 standard pressure levels which are interpolated to the Gaussian $p$-grid of 42 levels with the top level located at $0.8 \mathrm{hPa}$. In both cases the extrapolation below the surface requests zero under-surface winds and a constant geopotential. The vertical interpolation is carried out by using the cubic spline. The structure of four vertical modes of P21SP is presented in Fig. 2. The equivalent depths of displayed modes correspond to those of the four modes presented in Fig. 1b: $10 \mathrm{~km}$, about 40 $\mathrm{m}$, about $3.5 \mathrm{~m}$ and about $17 \mathrm{~cm}$. The circles along the zero line correspond to the standard pressure levels of the input data. It can be seen that levels are added in between the pressure levels by the interpolation; for example, six levels are added between $700 \mathrm{hPa}$ and $500 \mathrm{hPa}$ data levels. In the same pressure range, model-level data contain seven levels.

Although the two experiments with 91-level data use somewhat different truncation limits in the horizontal directions and the vertical direction, for the comparison of their energy distribution we sum up energy in the same modes: 70 vertical modes, 51 zonal wavenumbers (0:50) and 18 meridional modes $(0: 17)$ for each motion type. In the case of P21SP, 39 vertical modes are used while the horizontal truncations are the same as above. Energy percentages are rounded to the nearest integer as we found that the precision of our computations is up to $1 \%$ of wave energy which depends primarily on the number of the vertical modes included in the expansion. 


\section{Results}

Results of the three experiments are summarized in table Table 1 which shows the energy distribution in wave motions split among the balanced (R), the westward-propagating IG (WIG) and the eastward-propagating IG (EIG) waves. In order to keep consistence with the presentation in ZTAR2009 and TK1999 and at the same time, to respect the fact that many studies of the tropical circulations consider the mixed Rossby-gravity wave (MRG) as an IG mode, we show energy percentages in two ways: with the MRG mode included among the balanced modes (result denoted $\mathrm{R}+\mathrm{MRG}$ ) and the MRG mode included among the IG modes (denoted WIG+MRG).

There are two main results. First, the M91FD and M91SP experiments agree on the level of the IG energy in wave motions; the level is $10 \%$ to $12 \%$ depending on how we count the MRG mode. The experiment with the low vertical resolution, P21SP, indicates a lower energy content of the IG waves, 6\%. Secondly, all three experiments agree that the EIG energy exceeds the WIG energy; the difference is about (2-3)\% of the wave energy. It is due to the Kelvin modes, the most energetic IG mode of the atmosphere (Žagar et al. 2009b). We note that in the case when the MRG modes is counted among the WIG waves, the WIG+MRG and EIG energy level become the same in both M91FD and M91SP experiments. In conclusion, the comparison of the M91FD and M91SP confirms that the method for the solution of the vertical structure equation does not matter for the result as it has been expected. Table 2 further confirms the similarity of two solutions by comparing the energy content is several IG modes, in aadition to the Kelvin and MRG mode. The largest difference is for the MRG mode and it is most likely associated with the treatment of the top model levels (mesosphere) in the two numerical schemes. These experiments confirm that the tropical Kelvin mode makes about one-quarter of the global IG energy. Most of energy is in the zonal wavenumber 1 in the stratosphere (not shown). These energy percentages can for example be used for the verification of the coupled climate models which have largest uncertainties in the Tropics.

The comparison of the M91SP and P21SP experiments suggests that the difference between the percentages of energy estimated in ZTAR2009 and TK1996 is due to the input data density in the vertical direction. This result is in agreement with other studies that suggest that IG waves 
make a more significant contribution to the energy budgets of both atmosphere and oceans than it was previously thought. For example, the laboratory experiments at a low Rossby number found that about $9 \%$ of the flow energy was contributed by the IG waves emitted from the balanced flow (Williams et al. 2008). This suggests that our numbers may be the lower limit of the IG wave energy becaue the IG circulation described in our input data corresponds primarily to large scale in the Tropics (Žagar et al. 2009b).

Although we presented here just a single month of data, we believe the results are representative for longer dataset. In TK1996, nine years of winter ECMWF data were analyzed and compared with the NCEP and JMA analyses for the winter of 1988/1989. The study of TK1996 also showed that the interannual variability during the $\mathrm{NH}$ winter was not large. Our ongoing study with 20-year reanalysis data of ECMWF (ERA Interim) confirm the obtained percentages around $10 \%$ of the IG energy on large scales. The most variable IG mode is the Kelvin mode which has an annual cycle and a multi-year variability (not shown). We are currently extending our analysis to smaller horizontal scales to allow us to represent the IG circulation also in the mid-latitudes (Terasaki et al. 2011).

\section{Conclusions}

We have compared two methods for solving the vertical structure equation and two discretizations of the input data for the normal-mode function decomposition in order to address the differences in levels of IG energy reported in Tanaka and Kimura (1996, TK1996) and Žagar et al. (2009a, ZTAR2009). It is shown that the two methods lead to the same result provided the same input data are used; i.e. the level of IG energy in present-day global analyses is about $10 \%$ of the global wave energy. The eastward component of IG wave energy dominates over the westward IG wave energy due to the equatorial Kelvin wave which alone stands for about one-quarter of the global large scales IG wave energy.

1) *

Acknowledgments. The visit of N. Žagar to the University of Tsukuba was funded by the Center for Computational Sciences, by the Japan Society for the Promotion of Science and by 
the Slovenian Research Agency (ARRS). 


\section{References}

Andersson, E., P. Bauer, A. Beljaars, F. Chevallier, E. Holm, M. Janiskova, P. Kallberg, G. Kelly, P. Lopez, A. Mcnally, E. Moreau, A. J. Simmons, J.-N. Thepaut, and A. M. Tompkins, 2005: Assimilation and modeling of the atmospheric hydrological cycle in the ECMWF forecasting system. Bull. Amer. Meteor. Soc, , 86, 387-402.

Daley, R., 1983: Spectral characteristics of the ECMWF objective analysis system. ECMWF Tech. Rep. No. 40, 117 pp.

, 1991: Atmospheric data analysis. Cambridge University Press, Cambridge, UK, 460 pp.

Heckley, W. A., 1985: Systematic errors of the ECMWF operational forecasting model in tropical regions. Q. J. R. Meteorol. Soc., 111, 709-738.

Kasahara, A., 1976: Normal modes of ultralong waves in the atmosphere. Mon. Wea. Rev., 104, $669-690$.

_ 1984: The linear response of a stratified global atmosphere to a tropical thermal forcing. J. Atmos. Sci., 41, 2217-2239.

Kasahara, A. and K. Puri, 1981: Spectral representation of three-dimensional global data by expansion in normal mode functions. Mon. Wea. Rev., 109, 37-51.

Simmons, A. J. and A. Hollingsworth, 2002: Some aspects of the improvement in skill of numerical weather prediction. Q. J. R. Meteorol. Soc., 128, 647-677.

Staniforth, A., M. Beland, and J. Coté, 1985: An analysis of the vertical structure equation in sigma coordinates. Atmos.-Ocean, 23, 323-358.

Tanaka, H., 1985: Global energetics analysis by expansion into three-dimensional normal-mode functions during the FGGE winter. J. Meteor. Soc. Japan, 63, 180-200. 
Tanaka, H. and K. Kimura, 1996: Normal-mode energetics analysis and the intercomparison for the recent ECMWF, NMC, and JMA global analyses. J. Meteor. Soc. Japan, 74, 525-538.

Tanaka, H. and E. Kung, 1988: Normal-mode expansion of the general circulation during the FGGE year. J. Atmos. Sci., 45, 3723-3736.

Terasaki, K. and H. Tanaka, 2007: An analysis of the 3-D atmospheric energy spectra and interactions using analytical vertical structure functions and two reanalyses. J. Meteor. Soc. Japan, 85, 785-796.

Terasaki, K., H. Tanaka, and N. Žagar, 2011: Energy spectra of Rossby and gravity waves. SOLA, 11, 45-48.

Žagar, N., E. Andersson, and M. Fisher, 2005: Balanced tropical data assimilation based on a study of equatorial waves in ECMWF short-range forecast errors. Q. J. R. Meteorol. Soc., 131, 987-1011.

Žagar, N., N. Gustafsson, and E. Källén, 2004: Variational data assimilation in the tropics: the impact of a background error constraint. Q. J. R. Meteorol. Soc., 130, 103-125.

Žagar, N., J. Tribbia, J. L. Anderson, and K. Raeder, 2009a: Uncertainties of estimates of inertio-gravity energy in the atmosphere. Part I: intercomparison of four analysis datasets. Mon. Wea. Rev., 137, 3837-3857.

__ 2009b: Uncertainties of estimates of inertio-gravity energy in the atmosphere. Part II: large-scale equatorial waves. Mon. Wea. Rev., 137, 3858-3873.

—_ 2010: Corrigendum. Mon. Wea. Rev., 138, 2476-2477.

Williams, P. D., T. W. N. Haine, and P. L. Read, 2008: Inertia-gravity waves emitted from balanced flow: Observations, properties, and consequences. J. Atmos. Sci., 65, 3543-3556. 


\section{List of Figures}

1 Vertical eigen structures for the 91-level ECMWF model. (a) the finite-difference solution, (b) the spectral method solution. In each case, four vertical modes are presented: 1, 15, 30 and 60. Global mean vertical stability and temperature profiles are for July $2007 \ldots \ldots$. . . . . . . . . . . . . . . . . . 12

2 Vertical eigen structures based on the ECMWF 21 standard pressure-level data interpolated to the Gaussian $p$-grid of 42 points for the spectral method. The four vertical modes $(1,11,20$ and 35) have the same equivalent depths as the four modes presented in Fig. 1b. The round circles correspond to the locations of the standard pressure levels. . . . . . . . . . . . . . . . . . 13 

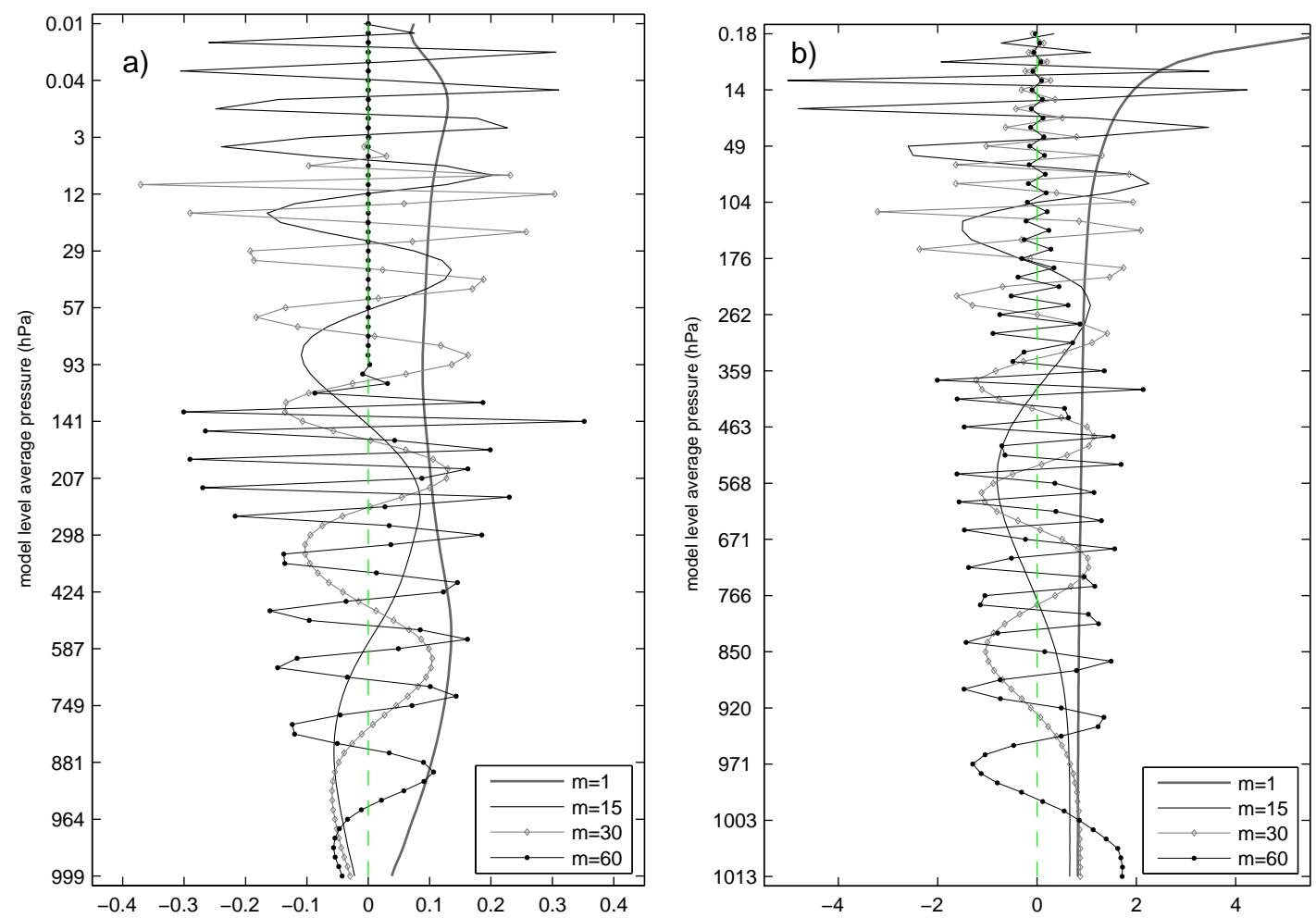

FIG. 1. Vertical eigen structures for the 91-level ECMWF model. (a) the finite-difference solution, (b) the spectral method solution. In each case, four vertical modes are presented: 1, 15, 30 and 60. Global mean vertical stability and temperature profiles are for July 2007. 


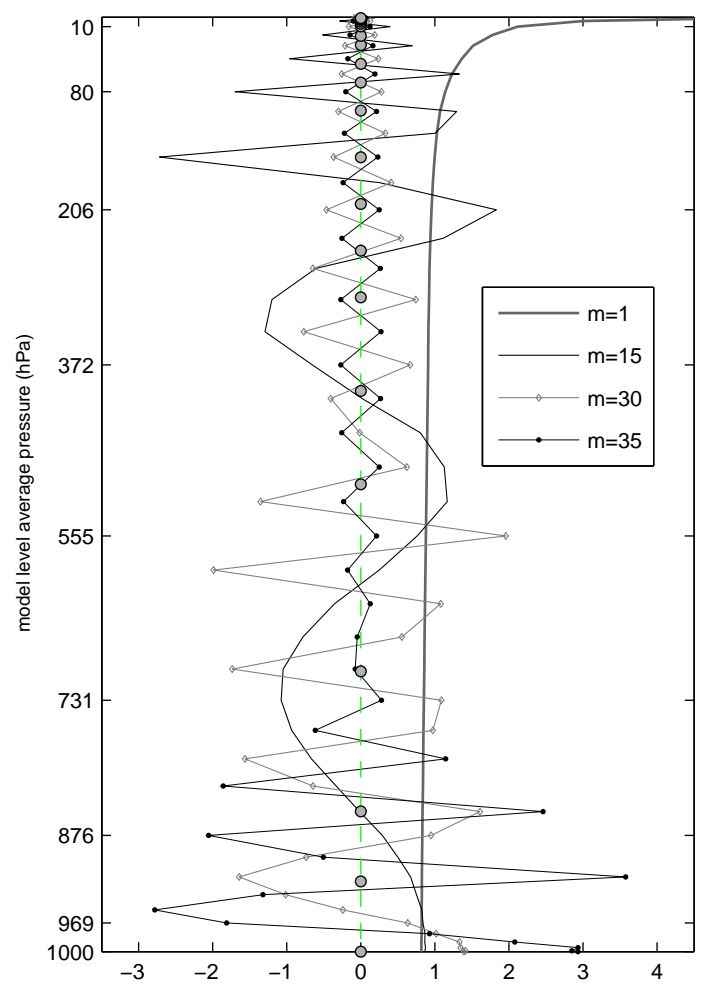

FIG. 2. Vertical eigen structures based on the ECMWF 21 standard pressure-level data interpolated to the Gaussian $p$-grid of 42 points for the spectral method. The four vertical modes (1, 11, 20 and 35) have the same equivalent depths as the four modes presented in Fig. 1b. The round circles correspond to the locations of the standard pressure levels. 


\section{List of Tables}

1 Distribution of energy among the balanced (R), the eastward inertio-gravity (EIG) and the westward inertio-gravity (WIG) motions as percentages of the total wave energy. Two cases are shown depending on whether the MRG mode is counted among the balanced modes $(\mathrm{R}+\mathrm{MRG})$ and among the WIG modes $(\mathrm{WIG}+\mathrm{MRG}) \ldots \ldots \ldots \ldots \ldots \ldots \ldots$

2 Energy percentages (\%) of different IG waves in the IG+MRG wave energy. The meridional mode index is denoted by $n \ldots \ldots$. . . . . . . . . . 16 
TABLE 1. Distribution of energy among the balanced (R), the eastward inertio-gravity (EIG) and the westward inertio-gravity (WIG) motions as percentages of the total wave energy. Two cases are shown depending on whether the MRG mode is counted among the balanced modes $(\mathrm{R}+\mathrm{MRG})$ and among the WIG modes (WIG+MRG).

\begin{tabular}{c|ccc} 
Experiment & R+MRG / R & EIG & WIG / (WIG+MRG) \\
\hline M91FD & $90 / 88$ & $6 / 6$ & $4 / 6$ \\
M91SP & $90 / 89$ & $7 / 7$ & $3 / 4$ \\
P21SP & $94 / 94$ & $4 / 4$ & $2 / 2$ \\
\hline
\end{tabular}


TABLE 2. Energy percentages (\%) of different IG waves in the IG+MRG wave energy. The meridional mode index is denoted by $n$.

\begin{tabular}{c|ccccc} 
Experiment & Kelvin & MRG & n=1 EIG & n=0 WIG & $n=1$ WIG \\
\hline M91FD & 22 & 18 & 6 & 8 & 4 \\
M91SP & 27 & 11 & 6 & 5 & 3 \\
\hline
\end{tabular}

